\title{
Theoretical study on the molecular and crystal structures of nitrogen trifluoride and it's adduct with $\mathrm{BF}_{3}$
}

\author{
HONGCHEN DU ${ }^{\mathrm{a}, \mathrm{b}, \mathrm{c}}$ \\ ${ }^{a}$ School of Science, Zhejiang A \& F University, Linan, 311300, China \\ bZhejiang Provincial Key Laboratory of Chemical Utilization of Forestry Biomass, \\ Zhejiang A \& F University, Lin'an, 311300, China \\ ${ }^{\mathrm{c}}$ Present address: Weifang University of Science and Technology, Jinguang Street 1299, \\ Shouguang, 262700, China \\ e-mail: duhongc@zafu.edu.cn; duhongchen123@126.com
}

MS received 30 April 2014; revised 30 October 2014; accepted 27 January 2015

\begin{abstract}
The molecular and crystal structure of the adduct $\mathrm{NF}_{3} \cdot \mathrm{BF}_{3}$ was studied computationally using density functional theory. It shows that the adduct exists in the form of a complex but is not ionic. The heats of formation in the gas and the condensed phase of the adduct are -1266.09 and $-1276.37 \mathrm{~kJ} \cdot \mathrm{mol}^{-1}$, respectively, which indicates that it is stable under atmospheric conditions. The crystal form belongs to $P 2_{1} / c$ space group. The calculated large band gap $\left(\Delta E_{\mathrm{g}}\right)$ of the crystal proves that it is stable. The conduction band (LUCO) is mainly contributed by the $p$ orbital of $\mathrm{N}$ atom and the valence band (HOCO) from the $p$ orbital of $\mathrm{F}$ atom.
\end{abstract}

Keywords. Molecular; crystal; structure; property; theoretical study.

\section{Introduction}

Molecular complexes containing boron trifluoride as a Lewis acid are known for many years. ${ }^{1}$ Nitrogen trifluoride $\left(\mathrm{NF}_{3}\right)$ is a colourless, toxic, odourless, nonflammable gas, which was first prepared in 1928 by Ruff, Fischer, and $\mathrm{Luft}^{2}$ by electrolyzing molten anhydrous ammonium difluoride in an electrically heated copper cell. The electrolytic process for the preparation of $\mathrm{NF}_{3}$ has been improved by a number of workers ${ }^{3-6}$ and has been patented. Nitrogen trifluoride can also be formed by direct fluorination of ammonia. It is a stable gas with strong oxidizing properties. It can be used as a potential oxidant for spacecraft propulsion. Decades ago, studies on the compound have been made: the infrared spectrum of $\mathrm{NF}_{3}$ has been reported by Bailey, Hale, and Thompson. ${ }^{7}$ In $1950, \mathrm{NF}_{3}$ was shown to have the $C_{3 \mathrm{v}}$ symmetry. ${ }^{8,9}$

Nitrogen trifluoride is mainly used for cleaning of PECVD chambers in high-volume production of liquid crystal displays and silicon-based thin film solar cells. $\mathrm{NF}_{3}$ has been considered environmentally preferable substitute for sulfur hexafluorine or perfluorocarbons such as hexafluoroethane. ${ }^{10}$ It proved to be far less reactive than other nitrogen trihalides, nitrogen trichloride, nitrogen tribromide and nitrogen triiodide, all of which are explosives. But explosion occurs when nitrogen trifluoride is mixed with ammonia, hydrogen, methane, ethylene, or carbon monoxide.

Understanding the nature of the structure-property relationship is of fundamental importance for further investigation even though there is limited information on the structure-property relationship, especially on the crystal structure of $\mathrm{NF}_{3}$ with $\mathrm{BF}_{3}$. In 1996, Ford et al. performed a theoretical study on the adduct, mainly its binding energies, using the ab initio method. ${ }^{11}$ This paper aims to study the molecular and crystal structure of the complex $\mathrm{NF}_{3} \cdot \mathrm{BF}_{3}$ and the properties.

\section{Computational details}

The title compound was optimized at the B3LYP/CCPVTZ level, and vibrational analysis was performed thereafter for the most stable conformer with the Gaussian 03 program package. ${ }^{12}$ Previous studies have shown that Beck's three-parameter nonlocal exchange functional along with the Lee-Yang-Parr nonlocal correlation functional (B3LYP) can figure out the accurate energy, molecular structure, and vibrational frequency, which are very close to the corresponding experimental results. ${ }^{13-16}$

The gas phase heat of formation $\left(\Delta_{\mathrm{f}} H_{\text {gas }}^{\circ}\right)$ was obtained using the following isodesmic reaction (1):

$$
\mathrm{NF}_{3}+\mathrm{BF}_{3}=\mathrm{NF}_{3} \cdot \mathrm{BF}_{3}
$$

The changes in enthalpy $\left(\Delta H_{298}^{\circ}\right)$ of the above reactions were evaluated using equation (2):

$$
\begin{aligned}
\Delta H_{298}^{\circ}= & \sum \Delta_{f} H_{298, \mathrm{P}}^{\circ}-\sum \Delta_{f} H_{298, \mathrm{R}}^{\circ}=\Delta E_{0} \\
& +\Delta E_{\mathrm{ZPE}}+\Delta H_{T}^{\circ}+\Delta \mathrm{nRT}
\end{aligned}
$$


where $\sum \Delta_{f} H_{298, \mathrm{P}}^{\circ}$ and $\sum \Delta_{f} H_{298, \mathrm{R}}^{\circ}$ are the sum of the heats of formation of the products and reactants, respectively; $\Delta E_{0}$ is the difference between the total energies of the products and the reactants at $0 \mathrm{~K} ; \Delta E_{\mathrm{ZPE}}$ is the difference between the zero-point vibrational energy of the products and the reactants; $\Delta H_{T}^{0}$ is the difference between the thermal correction from $0 \mathrm{~K}$ to $298 \mathrm{~K}$ of the products and the reactants, $\Delta n$ is the change in the quantity of gaseous substances, which is -1 here. In the reactions mentioned above, the experimental heats of formation of all reactants $\left(\mathrm{BF}_{3}, \mathrm{NF}_{3}\right)$ are known, ${ }^{17}$ the heats of formation of the adduct can then be obtained with the calculated $\Delta H_{298}^{\circ}$.

To find the possible molecular packings in crystal phase, empirical Dreiding force field and polymorph module in $\mathrm{MS}^{18}$ were used. Since most crystals belong to seven space groups (P21/c, P-1, P212121, P21, Pbca, $\mathrm{C} 2 / \mathrm{c}$, and Pna21) on the basis of statistical data, ${ }^{19-22}$ the global search was confined to these groups only. By analyzing the simulation trajectory of molecular packing within seven space groups, the structures were arranged in their ascending energies for each group and the one having the lowest energy was selected as the most possible packing with the corresponding space group. For solids, the local density approximation (LDA) and the generalized gradient approximation (GGA) are the most often used functionals. The GGA developed by Perdew, Burke, and Ernzerhof (PBE) is considered to be the standard for solids. ${ }^{23}$

These possible crystal structures were then refined with the DFT GGA-RPBE method and CASTEP module. ${ }^{24}$

\section{Results and Discussion}

\subsection{The choice of method}

To verify the accuracy, the B3LYP/CC-PVTZ method was performed to optimize nitrogen trifluoride first. We found that the point group at this level is $C_{3 \mathrm{~V}}$ (figure 1),

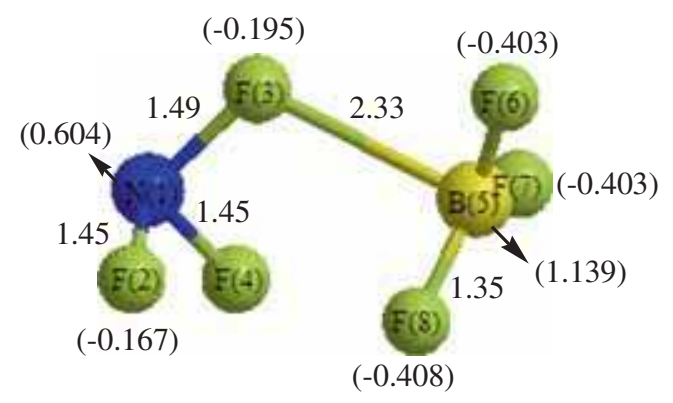

Figure 2. Structural parameters of the adduct obtained at the level of B3LYP/CC-PVTZ (bond lengths are in $\AA$, Mulliken charges(in brackets) in e).

which is consistent with the conclusion drawn from John et $a l .{ }^{8}$ the calculated infrared spectrum was then compared with the experimental results. ${ }^{25}$ Bailey claimed that the fundamental frequencies are located on $908 \mathrm{~cm}^{-1}$, which is also similar to the calculated result $\left(898 \mathrm{~cm}^{-1}\right)$, which indicates that the method B3LYP/CC-PVTZ is suitable for this study.

\subsection{Molecular structure}

Figure 2 shows the molecular structure of the adduct under B3LYP/CC-PVTZ level. It can be seen that in unit of $\mathrm{NF}_{3}$, the bond length of N-F are all similar, but N1-F3 is slightly larger, which denotes that N1-F3 is weaker. The bond length of B5-F3 is $2.33 \AA$, thus the bond is very weak. In addition, the negative charges on $\mathrm{F}(3)(-0.195 \mathrm{e})$ is also similar to $\mathrm{F}(2)$ and $\mathrm{F}(4)$, implying the same properties between $\mathrm{F}(3)$ and other $\mathrm{F}$ atoms. The total charges of $\mathrm{NF}_{3}$ and $\mathrm{BF}_{3}$ are nearly zero $(0.075 \mathrm{e}$ and $-0.075 \mathrm{e})$; therefore, the adduct exists in basically the complex form $\mathrm{NF}_{3} \cdot \mathrm{BF}_{3}$ but not ionic form.

The molecular electrostatic potential (MEP) is used commonly in analyzing molecular reactivity and is very useful since it provides information about local polarity due to the charge density distribution. After having chosen some sort of region to be visualized, a colour-coding convention is chosen to depict the MEP.
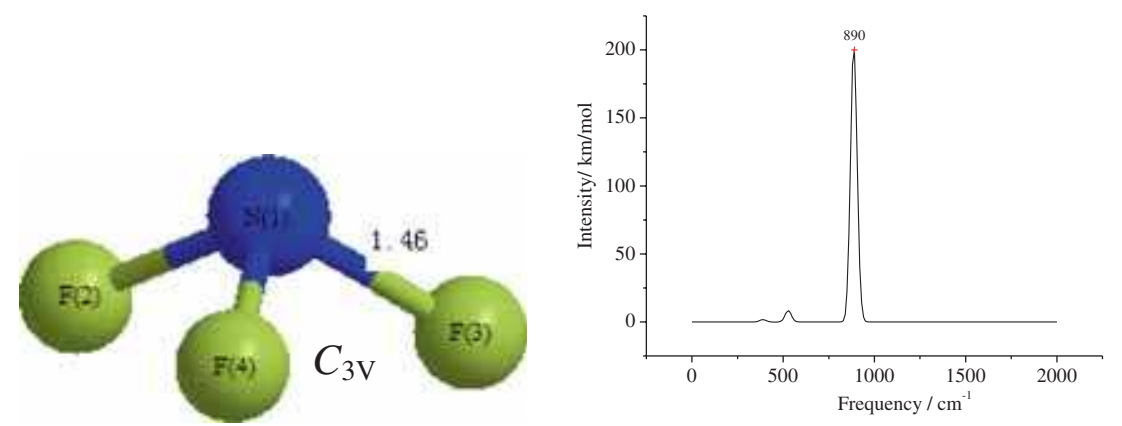

Figure 1. The calculated structure and infrared spectrum of $\mathrm{NF}_{3}$ under B3LYP/CC-PVTZ level. 


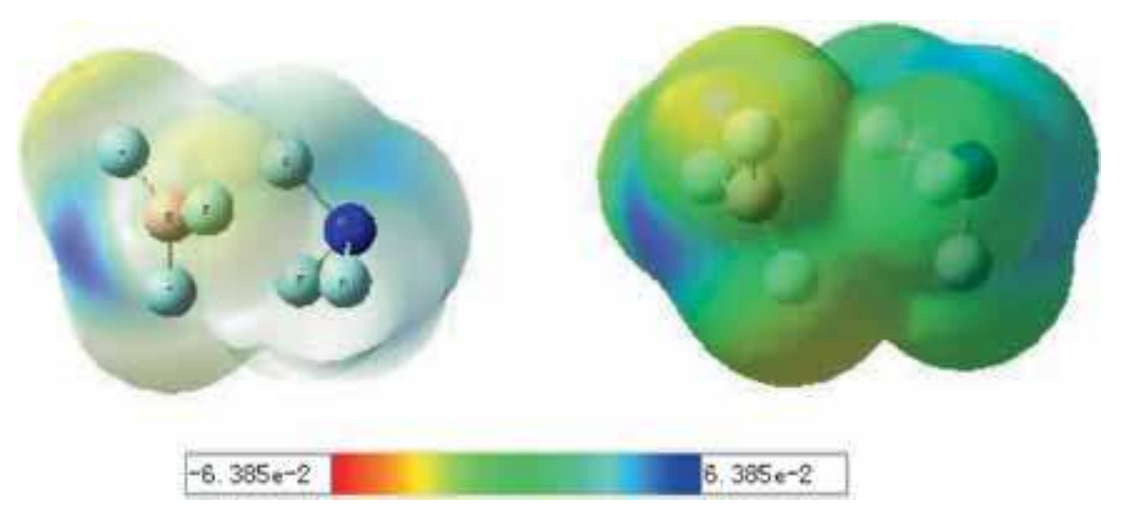

Figure 3. Molecular electrostatic potential (MEP) surface mapped onto 0.001 electron $/ \mathrm{bohr}^{3}$ contour of the electronic density for the title compound calculated at the B3LYP/-cc-PVTZ level Colour range: from red (negative) to blue (positive).

Figure 3 illustrates the MEP for the 0.001 electron/ bohr $^{3}$ isosurface of electron density at the B3LYP/CCPVTZ level for the $\mathrm{NF}_{3} \cdot \mathrm{BF}_{3}$. Red denotes the most negative potential and blue denotes the most positive potential. On inspection of the MEP for the title compound, the negative potentials appear to be distributed mostly on the fluorine atoms, and the positive ranges characterize at the centre of the skeleton, mainly on the nitrogen and boron atoms.

\subsection{Gas-phase heats of formation}

Heat of formation is usually taken as the indicator of the 'energy content' of a compound. It is very important to predict the heat of formation accurately. The gasphase heat of formation $\left(\Delta_{\mathrm{f}} H^{\circ}\right.$ gas $)$ has been estimated using the above isodesmic reaction. The experimental $\left(\Delta_{\mathrm{f}} H_{\text {gas }}^{\circ}\right.$ exp $)$ and predicted $\left(\Delta_{\mathrm{f}} H_{\text {gas }}^{\circ}\right.$ pre $) \Delta_{\mathrm{f}} H_{\text {gas }}^{\circ}$ using the isodesmic reaction of $\mathrm{NF}_{3} \cdot \mathrm{BF}_{3}$ are shown in table 1 . We can see that the $\Delta_{\mathrm{f}} H_{\text {gas }}^{\circ}$ of the adduct is large and negative $\left(-1266.09 \mathrm{~kJ} \cdot \mathrm{mol}^{-1}\right)$, which indicates the adduct is stable under atmospheric condition.

\subsection{Condensed-phase heats of formation}

For a crystal, the lattice energy ( $\left.E_{\text {latt }}\right)$ is important for predicting its structural and physicochemical properties, such as polymorphism and growth morphology. $E_{\text {latt }}$ can be calculated from the energy difference between the crystal $\left(E_{\text {crystal }}\right)$ and the isolated molecules $\left(E_{\text {molcule }}\right)$, that is

$$
E_{\text {latt }}=E_{\text {crystal }}-Z E_{\text {molecule }}
$$

where, $Z$ is the number of molecules in unit cell and is equal to 4 here. $E_{\text {latt }}$ is therefore the energy required for vaporizing a crystal and represents the strength of cohesion or interaction between molecules in the solid state. A negative value of $E_{\text {latt }}$ indicates an attractive intermolecular interaction in a crystal. The lattice energy of $\mathrm{NF}_{3} \cdot \mathrm{BF}_{3}$ obtained at DFT GGA/RPBE level is $-20.19 \mathrm{~kJ} \cdot \mathrm{mol}^{-1}$.

$E_{\text {latt }}$ was further used to evaluate the enthalpy of sublimation $\left(\Delta H_{\text {sub }}\right)$ using the following equation: ${ }^{26}$

$$
-\Delta H_{\mathrm{sub}}=E_{\mathrm{latt}}+E_{\mathrm{ZPE}}+\mathrm{ZRT}
$$

A rough estimation of the $\Delta H_{\text {sub }}$ is obtained by neglecting the $E_{\mathrm{ZPE}}$ term, and the solid-phase heat of formation $\left(\Delta_{\mathrm{f}} H^{\circ}\right.$ solid $)$ is then predicted from $\Delta_{\mathrm{f}}{H^{\circ}}_{\text {gas }}$ :

$$
\Delta_{\mathrm{f}} H_{\text {solid }}^{\circ}=\Delta_{\mathrm{f}} H_{\text {gas }}^{\circ}-\Delta H_{\text {sub }}
$$

The calculated $\Delta_{\mathrm{f}} H^{\circ}$ solid of $\mathrm{NF}_{3} \cdot \mathrm{BF}_{3}$ is -1276.37 $\mathrm{kJ} \cdot \mathrm{mol}^{-1}$.

Table 1. Total energies $\left(E_{0}\right)$ at the B3LYP/cc-PVTZ level and the gas-phase heats of formation.

\begin{tabular}{lccc}
\hline Compounds & $E_{0}$ (a.u.) & $\Delta_{\mathrm{f}} H_{\mathrm{gas}}^{\circ} \exp \left(\mathrm{kJ} \cdot \mathrm{mol}^{-1}\right)$ & $\Delta_{\mathrm{f}} H_{\mathrm{gas}}^{\circ \mathrm{pre}}\left(\mathrm{kJ} \cdot \mathrm{mol}^{-1}\right)$ \\
\hline $\mathrm{BF}_{3}$ & -324.6964 & -1135.60 & \\
$-\mathrm{NF}_{3}$ & -354.2227 & -132.09 & \\
$\mathrm{NF}_{3} \cdot \mathrm{BF}_{3}$ & -678.9213 & & -1266.09 \\
\hline
\end{tabular}




\subsection{Crystal structure}

As is known, among the 230 space groups, over $80 \%$ organic crystals belong to seven typical space groups based on the statistical data, which are $P 2_{1} / c, P 2_{1} 2_{1} 2_{1}$, $P$-1, $P$ bca, $C 2 / \mathrm{c}, P$ na2 ${ }_{1}$, and $P 2_{1} \cdot{ }^{27-29}$ The chosen force field methods (Universal and Dreiding) are commonly used to do a global search in the seven space groups mentioned above, ${ }^{30}$ and finally seven most stable polymorphs are obtained. The polymorph with the lowest energy will be recommended as the reasonable crystal from table 2 .

According to the principle, the most possible polymorph usually possesses lower energy. It can be concluded from table 2 that $\mathrm{NF}_{3} \cdot \mathrm{BF}_{3}$ tends to crystalline in $P 2_{1} / c$ from both Universal and Dreiding force field.

The density functional theory method DFT-GGARPBE was then performed to optimize the predicted packing $P 2_{1} / c$, the corresponding cell parameters are $a=6.93 \AA, b=13.44 \AA, c=5.79 \AA, \alpha=90.00^{\circ}$, $\beta=116.60^{\circ}, \gamma=90.00^{\circ}, \rho=1.72 \mathrm{~g} \cdot \mathrm{cm}^{-3}$ (figure 4).

\subsection{Band structure and density of states (DOS)}

When two valence electron atomic orbitals in a simple molecule such as hydrogen combine to form a

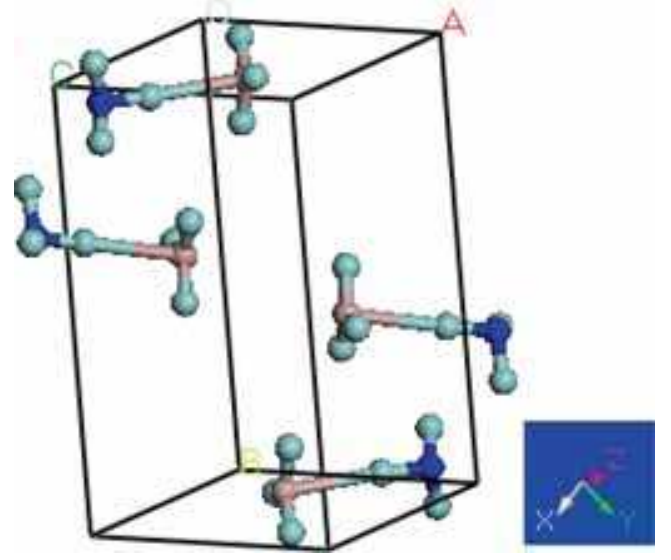

Figure 4. The optimized cell using DFT-GGA-RPBE method (molecular packing of the title compound in $\boldsymbol{P} \mathbf{2}_{\mathbf{1}} / \mathbf{c}$ space group).

chemical bond, two possible molecular orbitals result. One molecular orbital is lower in energy relative to the sum of the energies of the individual electron orbitals, and is referred to as the 'bonding' orbital. The other molecular orbital is raised in energy relative to the sum of the energies of the individual electron orbitals and is termed the 'anti-bonding' orbital.

If, as in the above one-dimensional schematic, a band is completely filled with electrons, and the band

Table 2a. Possible molecular packing for $\mathrm{NF}_{3} \cdot \mathrm{BF}_{3}$ in seven most possible space groups obtained from the universal force field.

\begin{tabular}{lccccccc}
\hline Space groups & $\boldsymbol{P 2} / \boldsymbol{c}$ & $P 2_{1} 2_{1} 2_{1}$ & $P-1$ & $P$ bca & $C 2 / \mathrm{c}$ & $P$ na2 ${ }_{1}$ & $P 2_{1}$ \\
\hline$Z$ & $\mathbf{4}$ & 4 & 2 & 8 & 8 & 4 & 2 \\
$E / \mathrm{kcal} / \mathrm{mol} /$ asym cell & $\mathbf{- 7 . 9 2}$ & -7.73 & -7.84 & -7.85 & -7.82 & -7.86 & -7.91 \\
$a / \AA$ & $\mathbf{4 . 4 9}$ & 12.11 & 5.91 & 12.01 & 12.93 & 12.40 & 4.82 \\
$b / \AA$ & $\mathbf{1 4 . 2 2}$ & 4.81 & 7.52 & 5.01 & 4.42 & 4.79 & 7.09 \\
$c / \AA$ & $\mathbf{6 . 5 9}$ & 7.27 & 4.85 & 13.94 & 16.79 & 7.05 & 6.14 \\
$\alpha{ }^{\circ}$ & $\mathbf{9 0 . 0 0}$ & 90.00 & 86.84 & 90.00 & 90.00 & 90.00 & 90.00 \\
$\beta /^{\circ}$ & $\mathbf{8 2 . 5 7}$ & 90.00 & 101.08 & 90.00 & 118.73 & 90.00 & 97.21 \\
$\gamma /{ }^{\circ}$ & $\mathbf{9 0 . 0 0}$ & 90.00 & 86.38 & 90.00 & 90.00 & 90.00 & 90.00 \\
$\rho / \mathrm{g} \cdot \mathrm{cm}^{-3}$ & $\mathbf{2 . 2 1}$ & 2.18 & 2.18 & 2.20 & 2.19 & 2.20 & 2.21 \\
& & & & & & &
\end{tabular}

Table 2b. Possible molecular packing for $\mathrm{NF}_{3} \cdot \mathrm{BF}_{3}$ in seven most possible space groups obtained from the Dreiding force field.

\begin{tabular}{lccccccc}
\hline Space groups & $\mathbf{P 2} / \boldsymbol{c}$ & $P 2_{1} 2_{1} 2_{1}$ & $P-1$ & $P$ bca & $C 2 / \mathrm{c}$ & $P$ na2 ${ }_{1}$ & $P 2_{1}$ \\
\hline$Z$ & $\mathbf{4}$ & 4 & 2 & 8 & 8 & 4 & 2 \\
$E / \mathrm{kcal} / \mathrm{mol} /$ asym cell & $\mathbf{- 6 . 0 7}$ & -5.80 & -5.98 & -5.69 & -5.95 & -5.89 & -5.93 \\
$a / \AA$ & $\mathbf{4 . 6 6}$ & 6.24 & 4.76 & 8.823 & 17.71 & 12.70 & 6.35 \\
$b / \AA$ & $\mathbf{1 4 . 5 6}$ & 8.82 & 11.35 & 15.61 & 4.79 & 4.94 & 7.30 \\
$c / \AA$ & $\mathbf{1 0 . 6 2}$ & 8.36 & 6.82 & 6.75 & 13.06 & 7.29 & 4.97 \\
$\alpha /^{\circ}$ & $\mathbf{9 0 . 0 0}$ & 90.00 & 128.56 & 90.00 & 90.00 & 90.00 & 90.00 \\
$\beta /{ }^{\circ}$ & $\mathbf{1 4 1 . 2 1}$ & 90.00 & 73.23 & 90.00 & 124.40 & 90.00 & 98.01 \\
$\gamma /{ }^{\circ}$ & $\mathbf{9 0 . 0 0}$ & 90.00 & 126.98 & 90.00 & 90.00 & 90.00 & 90.00 \\
$\rho / \mathrm{g} \cdot \mathrm{cm}^{-3}$ & $\mathbf{2 . 0 4}$ & 2.01 & 2.02 & 1.98 & 2.02 & 2.02 & 2.02 \\
\hline
\end{tabular}




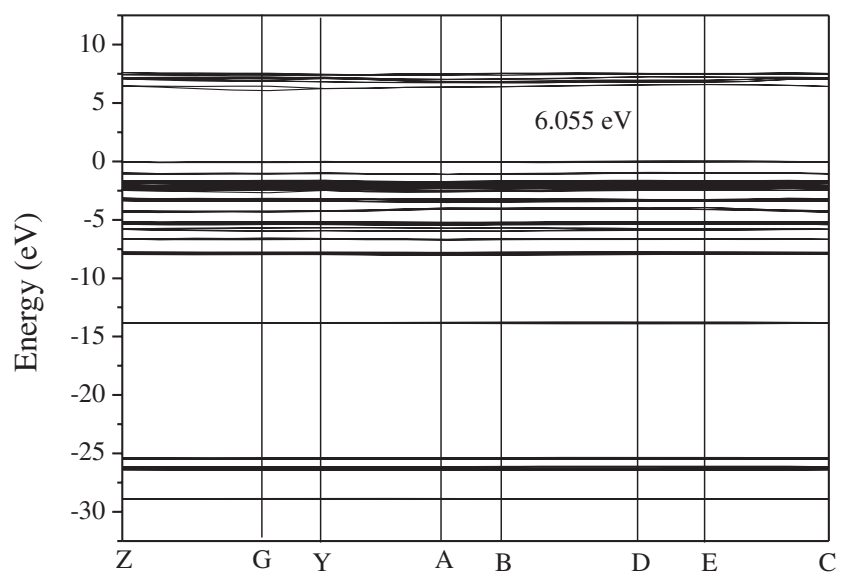

Figure 5. Energy band structure for $\mathrm{NF}_{3} \cdot \mathrm{BF}_{3}$. Note: The symbols $Z, G, Y, A, B, D, E, C$ means different symmetry directions of the Brillouin zone.

immediately above it is empty, the material has an energy band gap. This band gap is the energy difference between the highest occupied state in the valence band and the lowest unoccupied state in the conduction band. The material is a semiconductor if the band gap is relatively small or an insulator if the band gap is relatively large.

In principle, band gap $\left(\Delta E_{\mathrm{g}}\right)$ between the highest occupied crystal orbital (HOCO) and the lowest unoccupied crystal orbital (LUCO) can be used as a criterion to predict the sensitivity of energetic materials with a similar structure, and the smaller the $\Delta E_{\mathrm{g}}$, the easier the electron transits, and the molecule will be more active. This principle has been illustrated by many experimental results and is useful for both the ionic crystals ${ }^{31-34}$ and molecular crystals. ${ }^{35}$ Figure 5 presents the band of the predicted most probable packing using the GGARPBE method. It can be seen that the $\Delta E_{\mathrm{g}}$ of the title compound is large $(6.06 \mathrm{eV})$, which denotes that the crystal form of the adduct is also stable.

Density of state is a presentation of the band structure of a crystal. In condensed matter physics, the density of states (DOS) of a system describes the number of states per interval of energy at each energy level that are available to be occupied by electrons. Unlike isolated systems, like atoms or molecules in gas phase, the density distributions are not discrete like a spectral density but continuous. A high DOS at a specific energy level means that there are many states available for occupation. A DOS of zero means that no states can be occupied at that energy level. In general, a DOS is an average over the space and time domains occupied by the system. A better understanding of the band structure is its partial density of states (PDOS), in which DOS is projected on an atom-centered orbital, and PDOS

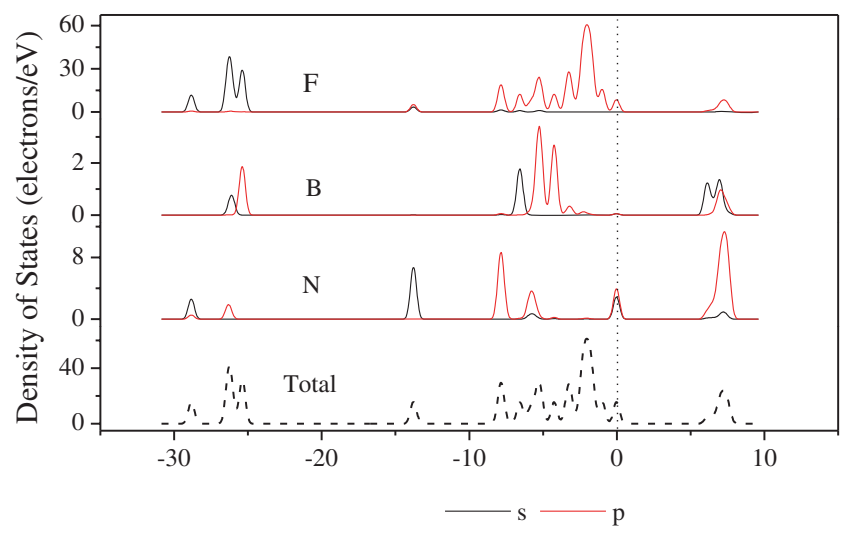

Figure 6. Total density of states (DOS) and partial DOS (PDOS) of the undoped $\mathrm{NF}_{3} \cdot \mathrm{BF}_{3}$ supercell. The Fermi energy is shown as a dashed vertical line.

can be used to investigate the constitution of energy bands. If the DOS of an undisturbed system is zero, the PDOS can locally be nonzero due to the presence of a local potential. PDOS further qualifies these results by resolving the contributions according to the angular momentum of the states. It is often useful to know whether the main peaks in the DOS are of $s, p$, or $d$ character.

Figure 6 gives the DOS and PDOS of the predicted crystal structure using the GGA-RPBE method, and the origin of the energy is taken to be the Fermi level (the vertical dotted line). It is noted that the conduction band (LUCO) is mainly contributed from the $p$ state of $\mathrm{N}$ atom and the valence band (HOCO) from the $p$ state of $\mathrm{F}$ atom.

\section{Conclusions}

The molecular and crystal structures of adduct $\mathrm{NF}_{3} \cdot \mathrm{BF}_{3}$ have a few differences. The adduct exists as a complex but not ionic. The heat of formation of the gas $\left(-1266.09 \mathrm{~kJ} \cdot \mathrm{mol}^{-1}\right)$ is slightly higher than the condensed phase $\left(-1276.37 \mathrm{~kJ} \cdot \mathrm{mol}^{-1}\right)$. Its crystal tends to be crystalline in $P 2_{1} / c$ space group, the optimized cell parameters are $a=6.93 \AA, b=13.44 \AA, c=5.79 \AA$, $\alpha=90.00^{\circ}, \beta=116.60^{\circ}, \gamma=90.00^{\circ}, \rho=$ $1.72 \mathrm{~g} \cdot \mathrm{cm}^{-3}$. The calculated band gap $(6.06 \mathrm{eV})$ proves that the crystal is stable, the conduction band (LUCO) is mainly contributed from the $p$ state of $\mathrm{N}$ atom and the valence band (HOCO) from the $p$ state of $\mathrm{F}$ atom.

\section{Acknowledgements}

This work was supported by Research and development Foundation (2012FR057, 2013FR019 and 2013FK026) of Zhejiang A \& F University. 
We are grateful for the technical support and computer time at the Sugon server of the computer centre of Nanjing University of Science \& Technology.

\section{References}

1. Greenwood N N and Martin R L 1954 Quart. Rev. 81

2. Ruff O, Fischer J and Luft F 1928 Z. Anorg. Chem. 172 417

3. Colburn C B, Parker C O and Stevenson K 1960 In Abstracts of Papers Presented at the 138th Meeting of the American Chemical Society (New York City)

4. Jarry R J and Miller H C 1956 J. Phys. Chem. 60 1412

5. Pierce L and Pace E L 1956 J. Chem. Phys. 221271

6. Ruff O 1929 Z. Angew. Chem. 42807

7. Bailey H 1937 J. Chem. Phys. 5274

8. John S 1950 Phys. Rev. 79513

9. Lu S 1950 J. Am. Chern. Soc. 721182

10. Reichardt H, Frenzel A and Schober K 2001 Microelectron. Eng. $\mathbf{5 6} 73$

11. Ford T A and Steele D 1996 J. Phys. Chem. 10019336

12. Frisch M J, Trucks G W and Pople J A 2003 Gaussian (Pittsburgh PA)

13. Zheng W, Wong N B, Liang X, Long X and Tian A 2004 J. Phys. Chem. A 108840

14. Wiberg K B J 2004 Comput. Chem. 251342

15. Li J 2008 Propell. Explos. Pyrot. 33443

16. Stephens P, Devlin F, Cheeseman J, Frisch M and Rosini C 2002 Org. Lett. 44595

17. Chase M W, Davies C A, Downey J R, Frurip D J, McDonald R A and Syverud A N 1985 JANAF Thermochemical Tables, 3rd ed. J. Phys. Chem. Ref. Data 14 Suppl. 1
18. Materials Studio 2008 In Version 4.4 (San Diego: Accelrys Software)

19. Wilson A J C 1988 Acta Crystallogr. Sect. A: Found Crystallogr. 44715

20. Mighell A D, Himes V L and Rodgers J R 1983 Acta Crystallogr. Sect A: Found Crystallogr. 39737

21. Srinivasan R 1992 Acta Crystallogr. Sect. A: Found Crystallogr. $\mathbf{4 8} 917$

22. Bau W H and Kassner D 1992 Acta Crystallogr. Sect. B: Struct. Sci. 48356

23. Haas P, Tran F and Blaha P 2011 Phys. Rev. B 83205117

24. Segall M D, Lindan P J D, Probert M J, Pickard C J, Hasnip P J, Clark S J and Payne M C J 2002 J. PhysCondens. Mat. 142717

25. Bailey C R, Hale J B and Thompson J W 2006 J. Chem. Phys. 5274

26. Giacovazzo C 1992 In Fundamentals of Crystallography (New York: Oxford University Press)

27. Wilson A J C 1988 Acta Crystallogr. Sect. A: Found Crystallogr. 44715

28. Srinivasan R 1992 Acta Crystallogr. Sect. A: Found Crystallogr. 48917

29. Baur W H and Kassner D 1992 Acta Crystallogr. Sect. B: Struct. Sci. 48356

30. Wang G, Shi C and Gong X 2009 J. Hazard. Mater. 169 813

31. Xiao H and Li Y 1996 In Banding and electronic structures of metal azides (Beijing: Science Press)

32. Zhu W, Xiao J and Xiao H 2006 J. Phys. Chem. B 110 9856

33. Zhu W, Xiao J and Xiao H 2006 Chem. Phys. Lett. 422 117

34. Xu X, Xiao H and Xiao J 2006 J. Phys. Chem. B 110 7203

35. Zhu W, Xiao J and Ji G 2007 J. Phys. Chem. B 1112715 